\title{
Editorial
}

\section{Advanced Nanomaterials for Green Growth}

\author{
${ }^{1}$ Vietnam National University, Hanoi, Vietnam \\ ${ }^{2}$ Lovely Professional University, Phagwara, India \\ ${ }^{3}$ Phenikaa University, Hanoi, Vietnam \\ Correspondence should be addressed to Thanh-Dong Pham; thanhdongpham080808@gmail.com
}

Thanh-Dong Pham $\mathbb{D}^{1},{ }^{1}$ Nguyen Van Noi, ${ }^{1}$ Ajit Kumar Sharma $\mathbb{D}^{2},{ }^{2}$ and Van-Duong Dao $\mathbb{D}^{3}$

Received 17 February 2020; Accepted 18 February 2020; Published 16 March 2020

Copyright (c) 2020 Thanh-Dong Pham et al. This is an open access article distributed under the Creative Commons Attribution License, which permits unrestricted use, distribution, and reproduction in any medium, provided the original work is properly cited.

Green and sustainable development is widely recognized as a concept of the modern society. This concept has appeared as a state of society where living conditions and resource use continue to meet human needs without undermining the integrity and stability of the natural system. Advanced nanomaterials can be applied to produce cleaner, more efficient, and valuable products to address our present environment-related concerns to a more sustainable future. Thus, the main goal of this issue is to highlight recent advances in synthesis and green applications of nanomaterials. The special issue contains twenty-eight selected research articles relating to nanomaterials. These reported nanomaterials present excellent performance and outstanding stability, have environmental benefits, and are cost-effective.

Most of the selected papers in the special issue reported the synthesis of nanomaterials applying for pollutant treatment. N. M. Phuong et al. reported novel activity of Fe$\mathrm{TiO}_{2} /$ Bent-Fe photocatalysts for removal of diazinon pesticides. T. T. V. Ha et al. presented synergistic adsorption and photocatalytic activity of $\mathrm{Ag}-\mathrm{ZnO} / \mathrm{GO}$ photocatalysts for extremely high removal of methylene blue. L. T. T. Thuy et al. reported high photocatalytic activity of $\mathrm{C} / \mathrm{Fe}$ codoped $\mathrm{TiO}_{2}$ coated on activated carbon for degradation of Rhodamine B (Rh B). H. Bibova et al. successfully synthesized $\mathrm{SiO}_{2} / \mathrm{TiO}_{2}$ composite coating on light substrates for photocatalytic decontamination of oxalic acid and methylene blue in water. L. T. T. Nguyen et al. successfully used the solution combustion method to synthesize $\mathrm{MgFe}_{2} \mathrm{O}_{4}$ nanoparticles for methylene blue dye degradation under visible light. N. T. Hanh et al. synthesized of Fe-modified biochar derived from rice straw to apply for arsenic removal.
H. X. Linh et al. reported that their synthesized red mudactivated graphite composites fast and effective in removing methylene blue from aqueous solutions. V. T. Pham et al. studied kinetics, isotherm, thermodynamics, and recyclability of exfoliated graphene-decorated $\mathrm{MnFe}_{2} \mathrm{O}_{4}$ nanocomposites for Congo red dye absorption. T. H. Le et al. successfully synthesized $\mathrm{MnO}_{2}$ nanoparticles on laterite for degradation of methylene blue. X. M. Pham et al. investigated the adsorption of $\mathrm{SO}_{2}$ and $\mathrm{NO}_{2}$ over porous $\mathrm{Fe}_{3} \mathrm{O}_{4}$ nanoparticles. D. T. C. Nguyen et al. successfully synthesized metal organic framework MIL-53(Fe) as an adsorbent for ibuprofen drug removal from aqueous solutions.

There are four selected papers in the special issue which presented application of nanomaterials as flame retardants. T. A. Nguyen et al. investigated mechanical properties and flame retardancy of epoxy resin/nanoclay/multiwalled carbon nanotube nanocomposites. H. T. Nhung et al. successfully used organoclay to modify expandable graphite/ polyurethane foam to use as a flame retardant and thermal insulator. V. Q. Tran et al. successfully used thermal shock combined with ball milling methods to prepare graphene nanoplatelets using flame retardant polymers. T. A. Nguyen et al. studied on fire resistance ability and mechanical properties of composites based on Epikote 240 epoxy resin and thermoelectric fly ash.

There are three selected papers in the special issue which reported catalytic activity of nanomaterials. S. T. Pham et al. reported that Al-incorporated SBA-15 highly efficiently catalyze cellulose conversion to 5 hydroxymethyl furfural (5HMF). T. H. T. Vu et al. successfully synthesized acidic heterogeneous catalysts with high stability based on 
graphene oxide/activated carbon composites for the esterification of lactic acid. M. D. Nguyen et al. reported enhancing activity of Pd-based/rGO modified by $\mathrm{Al}-\mathrm{Si}-\mathrm{Na}$ catalysts in ethanol electro-oxidation in an alkaline medium.

The special issue also presented other useful applications of nanomaterials. C. D. Trinh et al. used the wet chemical method to synthesize $\mathrm{YVO}_{4}$ : $\mathrm{Eu}^{3+}$ nanocrystals for luminescent security ink applications. T. T. P. Nguyen used carbon nanotubes and bismuth oxide to make electrodes for determination of metal concentration by the anodic stripping voltammetry method. T. L. Nguyen et al. successfully utilized platinum nanoflowers to modify electrodes to use as a sensitive sensor for simultaneous detection of lead and cadmium at trace levels. H. T. V. Nguyen et al. successfully used graphene oxide to prepare a hydrophilic polysulfone membrane.

These papers in the issue have been properly selected and concerned most recent progresses in the field of nanomaterials for green growth. The editors hope that the presented objectives of the special issue will be useful not only to working nanomaterial researchers but also to interested engineers as well as students and researchers in other fields. We offer many thanks for reading the enclosed papers and our best wishes for your future research.

\title{
Conflicts of Interest
}

The Guest Editor and Guest Co-Editors declare that there are no conflicts of interest or agreements with private companies, which will prevent us working impartially in the editorial process.

\section{Acknowledgments}

We are would like to deeply acknowledge all the authors for their contributions to the special issue and appreciate all the reviewers and related editorial board members for critical assessments and valuable comments to improve the quality of these papers.

\author{
Thanh-Dong Pham \\ Nguyen Van Noi \\ Ajit Kumar Sharma \\ Van-Duong Dao
}

